\title{
Distributed Construction of Trust Anchor with the Hyper-Powering Signature Scheme
}

\author{
Eisuke KOIZUMI*, Marika MINAGAWA and Hiroki SHIZUYA*
}

Graduate School of Information Sciences, Tohoku University, Sendai 980-8576, Japan

Received December 15, 2011; final version accepted May 22, 2012

\begin{abstract}
In this paper, we explore a strategy for recovering a PKI system without reconstructing the whole one when the trust anchor has been broken. Specifically, we propose two distributed signature schemes based on the hyperpowering discrete logarithm problem, which is a two-dimensional extension of the discrete logarithm problem. We show that these schemes are existentially unforgeable against the adaptively chosen message attack.
\end{abstract}

KEYWORDS: PKI, trust anchor, hyper-powering, distributed signature scheme, random oracle model

\section{Introduction}

Public key infrastructures (PKIs) are attracting much interest as foundations to achieve the security mechanisms such as confidence, integrity, authentication and non-repudiation. Using PKIs, one can apply public key cryptosystems to exchanging digitally signed information among parties which do not have a face to face interaction. In the process, a certification authority (CA) plays a critical role. Each party establishes a trust relationship with a CA, and the CA issues a digital certificate to each party. When a party communicates to another party, they establish trust to each other by using their certificates. Since the certificates are guaranteed to be valid by the CA, the parties can safely communicate as long as the trust of the CA is not infected.

In large-scale PKIs, generally, CAs work in cooperation with one another. Each CA uses a certificate issued by a "higher-level" CA in order to exchange an information between the CAs. Namely, the CAs lie on certificate chains to establish trust relationships. There are several trust models for the PKIs. In this paper, we focus on one of the widely used PKI structure, the hierarchical model, where all the CAs in a PKI form a rooted tree. The root CA certifies subordinate CAs, which in turn certify their subordinate CAs. Hence, in the hierarchical model, the root CA is a trust anchor of all the CAs and all the parties which form the PKI.

We should note that the hierarchical model involves a practical risk. Since the root CA takes the whole trust in the PKI, the security of the PKI collapses if the root CA is made unreliable by some attack. Consequently, all the CAs in the PKI lose their trust.

Yoshida et al. proposed an approach to overcoming the risk in [10]. The basic idea is that some of subordinate CAs deputize for the root CA in order to keep up the PKI when the root CA has lost its trust. In [10], they proposed the distributed RSA signature scheme, which is existentially unforgeable against the adaptively chosen message attack, as a means to restore the infected root CA without influencing the security and the function of the PKI. The theoretical background is very simple as intuitively described in the following. Let $(n, e)$ be a public key of the RSA scheme, and let $d$ be the corresponding private key. Assume that the root CA generates $n, e$ and $d$ which are different from the keys currently used. Then the root CA distributes $d_{i}(1 \leq i \leq k)$ to $k$ subordinate CAs so that $d \equiv d_{1}+\cdots+d_{k}(\bmod \varphi(n))$, where $\varphi$ is the Euler function. When the root CA lost its trust, it is taken over by $k$ CAs in a way that they cooperate to generate the signature $s$ of a message $m$ by $s_{i}=m^{d_{i}} \bmod n$ and $s=s_{1} \cdots s_{k} \bmod n$.

The method proposed in [10] strongly relies on the homomorphic property of the RSA signature scheme, and the construction itself is somewhat straightforward. However, it is not known so far whether one can achieve such a distributed construction with another widely used cryptographic primitives, such as the discrete logarithms.

In this paper, as an alternative to achieve the construction, we propose two distributed signature schemes based on the hyper-powering discrete logarithm problem [9], which is a two-dimensional extension of the discrete logarithm problem. In addition, we show that these schemes are existentially unforgeable against the adaptively chosen message attack.

This paper is organized as follows: In $\S 2$, we refer to the definition of the hyper-powering and some lemmas needed later. In $\S 3$, we propose two signature schemes based on the hyper-powering discrete logarithm problem, and state our

* Corresponding author. E-mail: koizumi@cite.tohoku.ac.jp; shizuya@cite.tohoku.ac.jp 
main result on their security. We also describe how to use these schemes in PKI. The proof of the result is given in $\$ 4-$ $\S 7$. We finally give concluding remarks in $§ 8$.

\section{The Hyper-Powering}

We refer to the definition of the hyper-powering in [9]. Let $p$ be a safe prime, that is, $p$ is a prime of the form $p=2 q+1$ for some prime $q$. Although we can define the hyper-powering over a generic finite field, we restrict ourselves to $\mathbb{Z}_{p}$ with safe prime $p$ for security reasons. Note that it is not proven that there are infinitely many safe primes. However, it is also widely believed that the set of safe primes, or equivalently the set of Sophie Germain primes is not finite [1]. Throughout this paper, we assume that there exist infinitely many safe primes. Let $B=\left(b_{i j}\right) \in M_{u}\left(\mathbb{Z}_{p}^{\times}\right)$ and $C=\left(c_{i j}\right) \in M_{u}\left(\mathbb{Z}_{p-1}\right)$, where $M_{u}\left(\mathbb{Z}_{p-1}\right)$ and $M_{u}\left(\mathbb{Z}_{p}^{\times}\right)$denote the ring of all $u \times u$ matrices over $\mathbb{Z}_{p-1}$ and the set of all $u \times u$ matrices over $\mathbb{Z}_{p}^{\times}$, respectively. The (right-handed) hyper-powering $B^{C}=D=\left(d_{i j}\right) \in M_{u}\left(\mathbb{Z}_{p}^{\times}\right)$is defined by

$$
d_{i j}=\prod_{l=1}^{u} b_{i l}^{c_{l j}} \bmod p .
$$

For any matrices $B=\left(b_{i j}\right)$ and $B^{\prime}=\left(b_{i j}^{\prime}\right)$ in $M_{u}\left(\mathbb{Z}_{p}^{\times}\right)$, we define a matrix $B \otimes B^{\prime}=B^{\prime \prime}=\left(b_{i j}^{\prime \prime}\right) \in M_{u}\left(\mathbb{Z}_{p}^{\times}\right)$by

$$
b_{i j}^{\prime \prime}=b_{i j} b_{i j}^{\prime} \bmod p \text {. }
$$

When $u=1$, the hyper-powering and the composition $\otimes$ coincide with the exponential law and the multiplication in $\mathbb{Z}_{p}^{\times}$, respectively. We immediately have the next lemma from the definition.

Lemma 2.1. Let $B, B^{\prime} \in M_{u}\left(\mathbb{Z}_{p}^{\times}\right)$and $C, C^{\prime} \in M_{u}\left(\mathbb{Z}_{p-1}\right)$. Then the following equations hold:

(i) $\left(B^{C}\right)^{C^{\prime}}=B^{C C^{\prime}}$,

(ii) $\left(B \otimes B^{\prime}\right)^{C}=B^{C} \otimes B^{\prime C}$, and

(iii) $B^{C} \otimes B^{C^{\prime}}=B^{C+C^{\prime}}$.

Let $B=\left(b_{i j}\right) \in M_{u}\left(\mathbb{Z}_{p}^{\times}\right)$, and assume that $1 \leq b_{i j} \leq p-1$ for any $i, j=1, \ldots, u$. Then one can naturally define a matrix $B^{*} \in M_{u}\left(\mathbb{Z}_{p-1}\right)$ by

$$
B^{*}=\left(b_{i j}^{*}\right)=\left(b_{i j} \bmod p-1\right) .
$$

In this paper, we propose two signature schemes based on a generalization of the discrete logarithm problem. The generalization is described in terms of the hyper-powering. The hyper-powering discrete logarithm problem of safe prime-type (SP-HDLP, for short) is to find a matrix $X \in M_{u}\left(\mathbb{Z}_{p-1}\right)$, for a given input $(p, g, A, Y)$, such that $Y=G^{X}$, where $p$ is a safe prime, $g$ is a generator of $\mathbb{Z}_{p}^{\times}, A=\left(a_{i j}\right) \in G L_{u}\left(\mathbb{Z}_{p-1}\right)$, the unit group of $M_{u}\left(\mathbb{Z}_{p-1}\right)$ and $G=g^{A}$ is a $u \times u$ matrix whose $(i, j)$-entry is $g^{a_{i j}}$. Note that the ordinary discrete logarithm problem of safe prime-type reduces to SP-HDLP by setting $u=1$ and $A=1$. Therefore, SP-HDLP is hard to solve as long as solving the ordinary discrete logarithm problem of safe-prime type is hard. An exact formulation of its hardness is given in the next section.

In the last of this section, we state the following lemma. This implies the existence and the uniqueness of the solution of SP-HDLP:

Lemma 2.2. Let $p, g$ and $A$ be as stated above. Then, for any $Y \in M_{u}\left(\mathbb{Z}_{p}^{\times}\right)$, there exists a unique matrix $X \in M_{u}\left(\mathbb{Z}_{p-1}\right)$ such that $Y=G^{X}$.

Proof. We first show the existence of a matrix $X$ satisfying $Y=G^{X}$. Note that $Y$ can be written of the form $Y=g^{Z}$ for some $Z \in M_{u}\left(\mathbb{Z}_{p-1}\right)$. Setting $X=A^{-1} Z$, we have $G^{X}=\left(g^{A}\right)^{A^{-1} Z}=g^{Z}=Y$.

We next show the uniqueness of $X$. If $Y=G^{X}=G^{X^{\prime}}$ holds for some $X, X^{\prime} \in M_{u}\left(\mathbb{Z}_{p-1}\right)$, then we have $A\left(X-X^{\prime}\right)=O$, where $O$ denotes the zero matrix. It follows from $A \in G L_{u}\left(\mathbb{Z}_{p-1}\right)$ that $X=X^{\prime}$.

\section{Statement of the Result}

In this section, we define two distributed signature schemes, and state our result on their security.

\subsection{Description of the Schemes}

Let $1^{m}$ denote a string of $m$ ones. For a $u \times u$ matrix $C=\left(c_{i j}\right)$ and a positive integer $m$, we write $C \bmod m=$ $\left(c_{i j} \bmod m\right)$, a $u \times u$ matrix whose $(i, j)$-entry is $c_{i j} \bmod m$.

Definition 3.1. The distributed hyper-powering signature scheme (DHS, for short) $\Sigma_{H}=\left(g_{H}, \wp_{H}, \mathcal{V}_{H}\right)$ consists of the following three algorithms:

- The key generation algorithm $g_{H}$ :

1. Input a tuple $\left(1^{k}, 1^{u}, 1^{n}\right)$.

2. Choose a safe prime $p$ of $k$ bits long, a generator $g$ of $\mathbb{Z}_{p}^{\times}$and $A \in G L_{u}\left(\mathbb{Z}_{p-1}\right)$.

3. For each $\mu=1, \ldots, n$, choose $X_{\mu} \in M_{u}\left(\mathbb{Z}_{p-1}\right)$, and compute $Y_{\mu}=G^{X_{\mu}}$, where $G=g^{A}$. 
4. Output $(p, g, A, Y)$ as a public key and $\left(X_{1}, \ldots, X_{n}\right)$ as the private key, where $Y=Y_{1} \otimes \cdots \otimes Y_{n}$.

- The signing algorithm $\wp_{H}$ :

1. Input a message $M \in\{0,1\}^{*}$ and a private key $\left(X_{1}, \ldots, X_{n}\right)$, where $\{0,1\}^{*}$ denotes the set of all strings over the finite alphabet $\{0,1\}$.

2. For each $\mu=1, \ldots, n$, choose $K_{\mu} \in M_{u}\left(\mathbb{Z}_{p-1}\right)$, and compute $R_{\mu}=G^{K_{\mu}}$.

3. Compute $H=h(M, R)$, where $R=R_{1} \otimes \cdots \otimes R_{n}$ and $h:\{0,1\}^{*} \times M_{u}\left(\mathbb{Z}_{p}^{\times}\right) \rightarrow M_{u}\left(\mathbb{Z}_{p-1}\right)$ is a hash function.

4. For each $\mu=1, \ldots, n$, set $S_{\mu}=X_{\mu} H-K_{\mu} R^{*} \bmod p-1$, where $R^{*}$ is the same manner as the Eq. (2.1).

5. Output a signature $\sigma=(R, H, S)$, where $S=S_{1}+\cdots+S_{n}$.

- The verification algorithm $\mathcal{V}_{H}$ :

1. Input a message $M$, a signature $\sigma$ and a public key $(p, g, A, Y)$.

2. Output 1 if $Y^{H}=R^{R^{*}} \otimes G^{S} \bmod p$, and 0 otherwise.

For each $\mu=1, \ldots, n, \sigma_{\mu}=\left(R, H, S_{\mu}\right)$ is called a partial signature. A pair $(M, \sigma)$ is said to be valid if $\mathcal{V}_{H}(M, \sigma)=1$.

If a signature $\sigma=(R, H, S)$ of a message $M$ is correctly computed by $\varsigma_{H}$, then we have

$$
\begin{aligned}
R^{R^{*}} \otimes G^{S} & =\left(R_{1} \otimes \cdots \otimes R_{n}\right)^{R^{*}} \otimes G^{S_{1}+\cdots+S_{n}} \\
& =R_{1}^{R^{*}} \otimes \cdots \otimes R_{n}^{R^{*}} \otimes G^{S_{1}} \otimes \cdots \otimes G^{S_{n}} \\
& =G^{K_{1} R^{*}} \otimes G^{S_{1}} \otimes \cdots \otimes G^{K_{n} R^{*}} \otimes G^{S_{n}} \\
& =G^{X_{1} H} \otimes \cdots \otimes G^{X_{n} H}=G^{\left(X_{1}+\cdots+X_{n}\right) H}=Y^{H},
\end{aligned}
$$

and hence, the pair $(M, \sigma)$ is valid.

This scheme is a generalization of the $(n, n)$ threshold signature scheme presented by Harn [6]. It was not shown in [6] that the scheme is provably secure. We note that the security, in the sense of Definition 3.4, of Harn's scheme immediately follows from our result.

We now describe how to use DHS in a PKI. Assume that there is a "trusted dealer" who manages the key distribution and generating signatures in the PKI. Let $\mathrm{CA}_{1}, \ldots, \mathrm{CA}_{n}$ denote all the subordinate CAs taking over the trust anchor from the root CA.

(i) The dealer chooses a safe prime $p$, a generator $g$ and a matrix $A$. Each $\mathrm{CA}_{\mu}$ randomly chooses a matrix $X_{\mu}$ as its private key, and computes $Y_{\mu}=G^{X_{\mu}}$. Then the dealer computes $Y$, and exhibits a public key $(p, g, A, Y)$.

(ii) When a party sends a message $M$ to the dealer, each $\mathrm{CA}_{\mu}$ randomly chooses $K_{\mu}$, and computes $R_{\mu}=G^{K_{\mu}}$. The dealer computes $R=R_{1} \otimes \cdots \otimes R_{n}$ and the hash value $H=h(M, R)$. Again, each $\mathrm{CA}_{\mu}$ computes $S_{\mu}=$ $X_{\mu} H-K_{\mu} R^{*}$. Then the dealer computes $S=S_{1}+\cdots+S_{n}$, and returns the signature $\sigma=(R, H, S)$ to the party.

(iii) Finally, using the verification algorithm, the party checks whether or not the signature is valid.

It is known that Harn's scheme is vulnerable to the rogue-key attack (e.g. [2]). DHS is also vulnerable to this attack. Hence, in order that DHS is to be secure, we need to assume that there exists a "trusted dealer" stated above or that all the subordinate CAs are honest.

We next define another distributed signature scheme as follows:

Definition 3.2. The distributed diagonal hyper-powering signature scheme (DDS, for short) $\Sigma_{D}=\left(g_{D}, \wp_{D}, \mathcal{V}_{D}\right)$ consists of the following three algorithms:

- The key generation algorithm $g_{D}$ :

1. Input a tuple $\left(1^{k}, 1^{u}, 1^{n}\right)$.

2. Choose a safe prime $p$ of length $k$, a generator $g$ of $\mathbb{Z}_{p}^{\times}$and $A \in G L_{u}\left(\mathbb{Z}_{p-1}\right)$, and set $G=g^{A}$.

3. For each $\mu=1, \ldots, n$, choose $X_{\mu} \in M_{u}\left(\mathbb{Z}_{p-1}\right)$, and compute $Y_{\mu}=G^{X_{\mu}}$.

4. Output $(p, g, A, \widetilde{Y})$ as a public key and $\left(X_{1}, \ldots, X_{n}\right)$ as the private key, where

$$
\widetilde{Y}=\left(\begin{array}{ccc}
Y_{1} & & 1 \\
& \ddots & \\
1 & & Y_{n}
\end{array}\right)
$$

- The signing algorithm $\varsigma_{D}$ :

1. Input a message $M \in\{0,1\}^{*}$ and a private key $\left(X_{1}, \ldots, X_{n}\right)$.

2. For each $\mu=1, \ldots, n$, choose $K_{\mu} \in M_{u}\left(\mathbb{Z}_{p-1}\right)$, and compute $R_{\mu}=G^{K_{\mu}}$.

3. For each $\mu=1, \ldots, n$, compute $H_{\mu}=h_{\mu}\left(M, R_{\mu}\right)$ and $S_{\mu}=X_{\mu} H_{\mu}-K_{\mu} R_{\mu}^{*} \bmod p-1$, where each $h_{\mu}:\{0,1\}^{*} \times M_{u}\left(\mathbb{Z}_{p}^{\times}\right) \rightarrow M_{u}\left(\mathbb{Z}_{p-1}\right)$ is a hash function.

4. Output a signature $\sigma=(\widetilde{R}, \widetilde{H}, \widetilde{S})$, where

$$
\widetilde{R}=\left(\begin{array}{ccc}
R_{1} & & 1 \\
& \ddots & \\
1 & & R_{n}
\end{array}\right), \quad \widetilde{H}=\left(\begin{array}{ccc}
H_{1} & & O \\
& \ddots & \\
O & & H_{n}
\end{array}\right), \quad \widetilde{S}=\left(\begin{array}{ccc}
S_{1} & & O \\
& \ddots & \\
O & & S_{n}
\end{array}\right) .
$$


- The verification algorithm $\mathcal{V}_{D}$ :

1. Input a message $M$, a signature $\sigma$ and a public key $(p, g, A, \widetilde{Y})$.

2. Output 1 if $\widetilde{Y}^{\tilde{H}}=\widetilde{R}^{*^{*}} \otimes \widetilde{G}^{S}$ holds, and 0 otherwise, where

$$
\widetilde{G}=\left(\begin{array}{ccc}
G & & 1 \\
& \ddots & \\
1 & & G
\end{array}\right) .
$$

For each $\mu=1, \ldots, n, \sigma_{\mu}=\left(R_{\mu}, H_{\mu}, S_{\mu}\right)$ is called a partial signature. A pair $(M, \sigma)$ is said to be valid if $\mathcal{V}_{D}(M, \sigma)=1$.

By the similar argument to the case of DHS, we see that a pair $(M, \sigma)$ of a message $M$ and a signature $\sigma$ is valid if $\sigma$ is correctly computed by $s_{D}$.

We note that DDS is resistant to the rogue-key attack since each piece $Y_{\mu}$ of the public key $\widetilde{Y}$ explicitly and independently appears in $\widetilde{Y}$.

Strictly speaking, the security parameter of these schemes is $k+u+n$. However, $k$ is set to be sufficiently larger than $u$ and $n$. Hence, one can regard $k$ as the security parameter. Then these schemes satisfy the conditions of generic digital signature schemes stated in [7].

\subsection{The Security Model}

In order to prove the security of DHS and DDS, we use the random oracle model formalized in Bellare and Rogaway [3]. In this model, a hash function is realized as an ideal random function. Namely, an oracle $\mathfrak{H}$ is called a random oracle if the following conditions hold:

(i) $\mathfrak{H}$ answers a random value for a new query which has not been queried yet, and

(ii) if the same query is asked twice, then $\mathfrak{H}$ answers the same value as has answered before.

We consider a model of a forger for DHS. The forger model for DDS can be formulated similar to that for DHS. Note that there are two crucial differences between our model and the canonical one defined in [4] and [7] since our schemes are "distributed" ones. First, a forger knows a part of a private key in advance. Second, the forger can get not only a signature but also partial signatures of any message. We now formulate a forger who attempts to break DHS as a probabilistic polynomial-time Turing machine (PPTM, for short) $\mathcal{F}$, which has the following properties:

(i) $\mathcal{F}$ takes as input a public key $(p, g, A, Y)$ and a part $\left(X_{1}, \ldots, X_{l}\right)$ of the private key which are output by $g_{H}\left(1^{k}, 1^{u}, 1^{n}\right)$, where $0 \leq l \leq n-1$.

(ii) $\mathcal{F}$ is allowed to access to a random oracle $\mathfrak{H}$. If $\mathcal{F}$ queries any pair $(M, R)$ of a message $M$ and $R \in M_{u}\left(\mathbb{Z}_{p}^{\times}\right)$, then $\mathfrak{H}$ answers an element $H \in M_{u}\left(\mathbb{Z}_{p-1}\right)$.

(iii) $\mathcal{F}$ is allowed to access to a signer oracle $\mathfrak{S}$. If $\mathcal{F}$ queries any pair $(M, I)$ of a message $M$ and a subset $I \subset\{l+1, \ldots, n\}$, then $\mathfrak{S}$ answers a set $\left\{\sigma_{\mu}\right\}_{\mu \in I}$ of partial signatures. Moreover, $\mathcal{F}$ can compute partial signatures $\sigma_{1}, \ldots, \sigma_{l}$ for any message $M$ by using $X_{1}, \ldots, X_{l}$.

(iv) Finally, $\mathcal{F}$ outputs a pair $(M, \sigma)$ of a message $M$ and a signature $\sigma$ or the symbol $\perp$, and halts, where $\perp$ is a special symbol not contained in $\{0,1\}^{*}$.

A forger $\mathcal{F}$ is said to succeed if $\mathcal{F}$ outputs a valid pair $(M, \sigma)$ such that $M \notin \mathcal{M}_{\mathfrak{S}}$, where $\mathcal{M}_{\mathfrak{S}}$ denotes the set of all messages which are queried to the signer oracle $\mathfrak{S}$ by $\mathcal{F}$.

We note that $\mathcal{F}$ is not allowed to do the rogue-key attack in the model above. The goal of $\mathcal{F}$ is to forge a valid signature for any fixed pair $\left((p, g, A, Y),\left(X_{1}, \ldots, X_{n}\right)\right)$ of public and private keys.

In the following two definitions, we use $\Sigma$ to designate $\Sigma_{H}$ or $\Sigma_{D}$.

Definition 3.3. Let $\mathcal{F}$ be a forger who attempts to break $\Sigma=(\mathscr{g}, \mathcal{S}, \mathcal{V})$, and let $0 \leq l \leq n-1$. $\mathcal{F}$ breaks $\Sigma$ with $\left(n, l ; t, q_{s}, q_{h}, \varepsilon\right)$ if, for any fixed random oracle $\mathfrak{H}$ and signer oracle $\mathfrak{S}$, the success probability of $\mathcal{F}$ is at least $\varepsilon$ under the following conditions:

(i) $\mathcal{F}$ is allowed to use a part $\left(X_{1}, \ldots, X_{l}\right)$ of the private key to compute partial signatures,

(ii) the running time of $\mathcal{F}$ is at most $t$, and

(iii) $\mathcal{F}$ is allowed to query $\mathfrak{H}$ and $\mathfrak{S}$ at most $q_{h}$ and $q_{s}$ times, respectively,

where the probability is taken over random tapes of $\mathcal{g}$ and $\mathcal{F}$ and random coin flipping of $\mathfrak{S}$.

Definition 3.4. The signature scheme $\Sigma$ is said to be $(n, l)$-existentially unforgeable against the adaptively chosen message attack $\left((n, l)\right.$-EUF-ACMA, for short) if the following condition holds: For any fixed polynomials $t, q_{s}$ and $q_{h}$ in $k$ and any forger who breaks $\Sigma$ with $\left(n, l ; t, q_{s}, q_{h}, \varepsilon\right), \varepsilon$ is negligible in $k$, that is,

for any polynomial $f$ in $k$.

$$
\lim _{k \rightarrow \infty} f(k) \varepsilon(k)=0
$$

The goal of this paper is to show the security of DHS and DDS as long as SP-HDLP is hard to solve. We exactly state the assumption. 
An adversary who tries to solve SP-HDLP is a PPTM $\mathcal{A}$ which has the following properties:

(i) $\mathcal{A}$ takes as input a tuple $(p, g, A, Y)$ of a safe prime $p$ of $k$ bits long, a generator $g$ of $\mathbb{Z}_{p}^{\times}$and matrices $A \in G L_{u}\left(\mathbb{Z}_{p-1}\right)$ and $Y \in M_{u}\left(\mathbb{Z}_{p}^{\times}\right)$, and

(ii) $\mathcal{A}$ outputs a matrix $X \in M_{u}\left(\mathbb{Z}_{p-1}\right)$ or $\perp$, and halts.

An adversary $\mathcal{A}$ is said to succeed in solving SP-HDLP if $\mathcal{A}$ outputs a matrix $X$ with $Y=G^{X}$.

Definition 3.5. Let $\mathcal{A}$ be an adversary who tries to solve SP-HDLP. $\mathcal{A}$ solves SP-HDLP with $(t, \varepsilon)$ if the running time of $\mathcal{A}$ is at most $t$ and the success probability of $\mathcal{A}$ is at least $\varepsilon$, where the probability is taken over the random tape of $\mathcal{A}$ and the random choices of $(p, g, A, Y)$.

Assumption 3.6 (SP-HDLP assumption). For any fixed polynomial $t$ in $k$ and any adversary $\mathcal{A}$ who solves SP$H D L P$ with $(t, \varepsilon), \varepsilon$ is negligible in $k$.

We are now ready to state our main result.

\subsection{Main Result}

Theorem. (i) DHS is (n,0)-EUF-ACMA under the SP-HDLP assumption.

(ii) For any $0 \leq l \leq n-1, D D S$ is $(n, l)$-EUF-ACMA under the SP-HDLP assumption.

The proof of the theorem is given in the next section.

\section{Proof of the Theorem}

The following lemma plays an important role in the proof of the theorem:

Lemma A. (i) Assume that there exists a forger who breaks DHS with $\left(n, 0 ; t, q_{s}, q_{h}, \varepsilon\right)$ and that $\varepsilon \geq$ $10\left(q_{s}+1\right)\left(q_{s}+q_{h}\right) / 2^{k}$. Then there exists an adversary who solves SP-HDLP with $\left(t^{\prime}, \varepsilon^{\prime}\right)$, where

$$
t^{\prime}=2 t+2\left(q_{h}+q_{s}+1\right) O\left(n k^{3} u^{4}\right), \quad \frac{3 \varepsilon^{2}}{400 q_{h}} \leq \varepsilon^{\prime} .
$$

(ii) Let $0 \leq l \leq n-1$. Assume that there exists a forger who breaks DDS with $\left(n, l ; t, q_{s}, q_{h}, \varepsilon\right)$ and that $\varepsilon \geq 10\left(q_{s}+1\right)\left(q_{s}+q_{h}\right) / 2^{k}$. Then there exists an adversary who solves SP-HDLP with $\left(t^{\prime}, \varepsilon^{\prime}\right)$, where

$$
t^{\prime}=2 t+2\left(q_{h}+q_{s}+1\right) O\left(n k^{3} u^{4}\right), \quad \frac{3 \varepsilon^{2}}{400 q_{h}} \leq \varepsilon^{\prime} .
$$

We prove Lemma A in the next section, and first give the proof of the theorem assuming that Lemma A holds.

Suppose that there exist polynomials $t, q_{s}$ and $q_{h}$ in $k$ and a forger $\mathcal{F}_{H}$ who breaks DHS with $\left(n, 0 ; t, q_{s}, q_{h}, \varepsilon\right)$ for some function $\varepsilon$. When $\varepsilon<10\left(q_{s}+1\right)\left(q_{s}+q_{h}\right) / 2^{k}$, (i) of the theorem immediately follows since $\varepsilon$ is negligible in $k$. Assume that $\varepsilon \geq 10\left(q_{s}+1\right)\left(q_{s}+q_{h}\right) / 2^{k}$. Using (i) of Lemma A, we can construct an adversary $\mathscr{A}_{H}$ who solves SPHDLP with

$$
\left(2 t+2\left(q_{h}+q_{s}+1\right) O\left(n k^{3} u^{4}\right), \frac{3 \varepsilon^{2}}{400 q_{h}}\right) .
$$

By Assumption 3.6, the success probability $3 \varepsilon^{2} /\left(400 q_{h}\right)$ of $\mathcal{A}_{H}$ is negligible in $k$. We therefore have

$$
f_{1}(k)(\varepsilon(k))^{2}=\frac{400 q_{h}(k) f_{1}(k)}{3} \cdot \frac{3(\varepsilon(k))^{2}}{400 q_{h}(k)} \longrightarrow 0
$$

as $k \rightarrow \infty$ for any polynomial $f_{1}$. This shows that $(\varepsilon(k))^{2}$ is negligible. We also have

$$
\left(f_{2}(k) \varepsilon(k)\right)^{2}=\left(f_{2}(k)\right)^{2}(\varepsilon(k))^{2} \longrightarrow 0
$$

as $k \rightarrow \infty$ for any polynomial $f_{2}$. Hence, we see that $\varepsilon$ is also negligible, and (i) of the theorem follows. We can similarly show (ii) of the theorem.

\section{Proof of Lemma $A$}

We return to Lemma A. The proof of the lemma is based on Lemmas 2 and 4 in [7]. Informally, these lemmas state that running a forger several times with different random oracles, one can obtain two "appropriate" signatures $\sigma$ and $\sigma$ ' of the same message $M$ with some probability. By using these lemmas, Pointcheval and Stern proved, in [7], the security of the Schnorr signature scheme [8] and the modified ElGamal signature scheme [7]. We prove Lemma A by arguments similar to the proof of lemmas in [7].

In order to construct an adversary described in Lemma A, we need to simulate properly the interactions which the forger should have with a random oracle and a signer oracle. In addition, those simulations should be indistinguishable from the interactions with the real oracles. 
We first show that one can properly simulate the interactions.

Lemma 5.1. Let $(p, g, A)$ be a tuple of a safe prime $p=2 q+1$, a generator $g$ of $\mathbb{Z}_{p}^{\times}$and a matrix $A=$ $\left(a_{i j}\right) \in G L_{u}\left(\mathbb{Z}_{p-1}\right)$. Then, for any matrix $Y \in M_{u}\left(\mathbb{Z}_{p}^{\times}\right)$, one can compute a matrix $C \in M_{u}\left(\mathbb{Z}_{2}\right)$ such that $C=X \bmod 2$ for a matrix $X \in M_{u}\left(\mathbb{Z}_{p-1}\right)$ satisfying $Y=G^{X}$, where $G=g^{A}$.

Proof. Let $X \in M_{u}\left(\mathbb{Z}_{p-1}\right)$ be the matrix satisfying $Y=G^{X}$. We consider the following procedure:

Procedure Find- $C$.

(F-1) Input a tuple $(p, g, A, Y)$.

(F-2) Compute $Y^{q I_{u}}$, where $I_{u}$ is the identity matrix of degree $u$.

(F-3) For each pair $(i, j)$ of indices $i, j=1, \ldots, u$, solve the following system (5.1) of linear equations with respect to $c_{i j} \in \mathbb{Z}_{2}$ :

$$
q^{2} \sum_{\zeta=1}^{u} a_{i \zeta} c_{\zeta j}= \begin{cases}0 \bmod 2 & \text { if } y_{i j}^{q}=1 \bmod p \\ q \bmod 2 & \text { if } y_{i j}^{q}=-1 \bmod p .\end{cases}
$$

(F-4) Output the matrix $C=\left(c_{i j}\right)$.

Since $A \bmod 2 \in G L_{u}\left(\mathbb{Z}_{2}\right)$, an output of the procedure is uniquely determined. We show that $C=X$ mod 2 . Note that there exist matrices $B \in M_{u}\left(\mathbb{Z}_{q}\right)$ and $C^{\prime} \in M_{u}\left(\mathbb{Z}_{2}\right)$ such that $X=2 B+q C^{\prime} \bmod p-1$. We have $Y^{q I_{u}}=G^{2 q B+q^{2} C^{\prime}}=$ $G^{q^{2} C^{\prime}}$. Since $\left(y_{i j}^{q}\right)^{2}=1 \bmod p$ for all indices $i, j=1, \ldots, u, y_{i j}^{q}$ is equal to $1 \bmod p$ or $-1 \bmod p$. We also have $1 \bmod$ $p=g^{0} \bmod p$ and $-1 \bmod p=g^{(p-1) / 2} \bmod p=g^{q} \bmod p$.

On the other hand, we have $y_{i j}^{q}=\left(G^{q^{2} C^{\prime}}\right)_{i j}=g^{q^{2}\left(a_{i 1} c_{1 j}^{\prime}+\cdots+a_{i u} c_{u j}^{\prime}\right)}$. Hence the matrix $C^{\prime}$ is a solution of (5.1), and we have $C^{\prime}=C=X \bmod 2$.

Lemma 5.2. Let $(p, g, A)$ be as in Lemma 5.1, and let $n$ be any positive integer. Then, for any given $Y \in M_{u}\left(\mathbb{Z}_{p}^{\times}\right)$and $X_{1}, \ldots, X_{n-1} \in M_{u}\left(\mathbb{Z}_{p-1}\right)$, one can produce a valid signature $(R, H, S)$ of DHS without using the matrix $X_{n}$.

Proof. Set $Y_{n}=Y \otimes G^{-\left(X_{1}+\cdots+X_{n-1}\right)}$. We can compute $X_{n}^{(2)}=X_{n} \bmod 2$ such that $Y_{n}=G^{X_{n}}$ by Lemma 5.1. Consider the following procedure:

Procedure Simulation.

(S-1) Input a tuple $\left(p, g, A, Y, X_{1}, \ldots, X_{n-1}, X_{n}^{(2)}\right)$.

(S-2) Choose $K_{1}, \ldots, K_{n-1} \in M_{u}\left(\mathbb{Z}_{p-1}\right), E_{n}, V_{n} \in M_{u}\left(\mathbb{Z}_{q}\right)$ and $W_{n} \in M_{u}\left(\mathbb{Z}_{2}\right)$ randomly.

(S-3) Compute $R_{n}=G^{2 E_{n}} \otimes Y_{n}^{2 V_{n}} \otimes G^{q W_{n}} \bmod p$ and $R_{\mu}=G^{K_{\mu}}$ for each $\mu=1, \ldots, n-1$, and set $R=$ $R_{1} \otimes \cdots \otimes R_{n} \bmod p$.

(S-4) Compute $H^{(q)}=2 V_{n} R^{*} \bmod q$, and choose $H^{(2)} \in M_{u}\left(\mathbb{Z}_{2}\right)$ randomly. Then set $H=H^{(q)}+q H^{(2)} \bmod p-1$, and compute $S_{\mu}=X_{\mu} H-K_{\mu} R^{*} \bmod p-1$ for each $\mu=1, \ldots, n-1$.

(S-5) Compute $S_{n}^{(2)}=X_{n}^{(2)} H^{(2)}-W_{n} R^{*} \bmod 2$ and $S_{n}^{(q)}=-2 E_{n} R^{*} \bmod q$. Then set $S_{n}=S_{n}^{(q)}+q S_{n}^{(2)} \bmod p-1$.

(S-6) Output the tuple $\left(R, H, S_{1}, \ldots, S_{n}\right)$.

We now show that $(R, H, S)$ is a valid signature, where $S=S_{1}+\cdots+S_{n}$. Set $K_{n}=2\left(E_{n}+X_{n} V_{n}\right)+q W_{n} \bmod p-1$. We have

$$
K_{n} R^{*}+S_{n} \bmod 2=W_{n} R^{*}+X_{n}^{(2)} H^{(2)}-W_{n} R^{*} \bmod 2=X_{n} H \bmod 2
$$

and

$$
\begin{aligned}
K_{n} R^{*}+S_{n} \bmod q & =2\left(E_{n}+X_{n} V_{n}\right) R^{*}-2 E_{n} R^{*} \bmod q \\
& =X_{n}\left(2 V_{n} R^{*}\right) \bmod q=X_{n} H \bmod q .
\end{aligned}
$$

It follows from these equations and (S-4) that

$$
S=\left(X_{1}+\cdots+X_{n}\right) H-\left(K_{1}+\cdots+K_{n}\right) R^{*} \bmod p-1 .
$$

Hence, we have $Y^{H}=G^{S} \otimes R^{R^{*}} \bmod p$. This proves the lemma.

We next show that Procedure Simulation constructed in the proof of Lemma 5.2 is indistinguishable from real interactions. We describe an exact formulation of the indistinguishability of the simulation.

Let $(p, g, A)$ be as in Lemma 5.1, and let $X_{1}, \ldots, X_{n} \in M_{u}\left(\mathbb{Z}_{p-1}\right)$. We write $\Omega_{1}=\left(M_{u}\left(\mathbb{Z}_{p-1}\right)\right)^{n+1}, \Omega_{2}=$ $\left(M_{u}\left(\mathbb{Z}_{p-1}\right)\right)^{n-1} \times\left(M_{u}\left(\mathbb{Z}_{q}\right)\right)^{2} \times\left(M_{u}\left(\mathbb{Z}_{2}\right)\right)^{2}$ and $\Omega_{3}=\left(M_{u}\left(\mathbb{Z}_{p}^{\times}\right)\right)^{n} \times\left(M_{u}\left(\mathbb{Z}_{p-1}\right)\right)^{n+1}$. We define a map $F_{1}: \Omega_{1} \rightarrow \Omega_{3}$ by

$$
F_{1}\left(K_{1}, \ldots, K_{n}, H\right)=\left(G^{K_{1}}, \ldots, G^{K_{n}}, H, S_{1}, \ldots, S_{n}\right),
$$

where $R=G^{K_{1}} \otimes \cdots \otimes G^{K_{n}}$ and $S_{\mu}=X_{\mu} H-K_{\mu} R^{*}$ for each $\mu=1, \ldots, n$. We also define a map $F_{2}: \Omega_{2} \rightarrow \Omega_{3}$ by

$$
F_{2}\left(K_{1}, \ldots, K_{n-1}, E_{n}, V_{n}, W_{n}, H^{(2)}\right)=\left(G^{K_{1}}, \ldots, G^{K_{n-1}}, R_{n}, H, S_{1}, \ldots, S_{n}\right),
$$

where

$$
\begin{gathered}
R_{n}=G^{2\left(E_{n}+X_{n} V_{n}\right)+q W_{n}}, \quad R=G^{K_{1}} \otimes \cdots \otimes G^{K_{n-1}} \otimes R_{n}, \\
H=2 V_{n} R^{*}+q H^{(2)} \bmod p-1, \quad S_{n}=-2 E_{n} R^{*}+q\left(X_{n} H-W_{n} R^{*}\right) \bmod p-1
\end{gathered}
$$


and $S_{\mu}=X_{\mu} H-K_{\mu} R^{*}$ for each $\mu=1, \ldots, n-1$. Set $\mathscr{S}_{i}=F_{i}\left(\Omega_{i}\right)$ for $i=1,2$. Note that $\mathscr{S}_{1}$ is the set of all tuples produced by a random oracle and a signer oracle and that $\mathscr{S}_{2}$ is the set of all tuples produced by Procedure Simulation. Lemma 5.2 implies that $\mathscr{S}_{2} \subset \mathscr{S}_{1}$. We show that $\mathscr{S}_{1}$ and $\mathscr{S}_{2}$ are indistinguishable. More precisely, we prove the following lemma:

Lemma 5.3. $\mathscr{S}_{1}$ and $\mathscr{S}_{2}$ are statistically indistinguishable, that is, the value

$$
d\left(\Omega_{1}, \Omega_{2}\right)=\sum_{\sigma \in \mathscr{S}_{1}}\left|\operatorname{Pr}_{\omega_{1} \in \Omega_{1}}\left[F_{1}\left(\omega_{1}\right)=\sigma\right]-\operatorname{Pr}_{\omega_{2} \in \Omega_{2}}\left[F_{2}\left(\omega_{2}\right)=\sigma\right]\right|
$$

is negligible in $k$, where the probability $\operatorname{Pr}_{\omega_{i} \in \Omega_{i}}$ is taken over random choices of $\omega_{i} \in \Omega_{i}$ for $i=1,2$.

The proof of this lemma is given in the next section, and in the rest of this section, we prove Lemma A. We first prove (i) of the lemma.

Let $\mathcal{F}_{H}$ be a forger who breaks DHS with $\left(n, 0 ; t, q_{s}, q_{h}, \varepsilon\right)$. We construct a PPTM $\mathcal{A}_{H}$ as follows:

(1) $\mathcal{A}_{H}$ takes as input a tuple $(p, g, A, Y)$.

(2) Choose $X_{1}, \ldots, X_{n-1} \in M_{u}\left(\mathbb{Z}_{p-1}\right)$ randomly, and set $Y_{n}=Y \otimes G^{-\left(X_{1}+\cdots+X_{n-1}\right)}$. Then execute Procedure Find- $C$ on input $\left(p, g, A, Y_{n}\right)$, and set $X_{n}^{(2)}=C$.

(3) Set a counter $\alpha$ by 0.

(4) Run $\mathcal{F}_{H}$ on input $(p, g, A, Y)$ with some random tape $\omega$.

(5) While running $\mathcal{F}_{H}$, if $\mathcal{F}_{H}$ queries $\mathfrak{S}$ or $\mathfrak{H}$, then go to Step (5-1) or (5-2).

(5-1) When $\mathcal{F}_{H}$ asks a pair $(M, I)$ of a message $M$ and a subset $I \subset\{1, \ldots, n\}$ to $\mathfrak{S}$, execute the following procedure:

(i) Increase $\alpha$ by one, and set $M_{\alpha}=M$. Then add $M_{\alpha}$ to the message list $\mathcal{M}_{\mathfrak{S}}$.

(ii) Execute Procedure Simulation on input $\left(p, g, A, Y, X_{1}, \ldots, X_{n-1}, X_{n}^{(2)}\right)$.

(iii) Set $\left(R_{\alpha}, H_{\alpha}, S_{\mu, \alpha}\right)=\left(R, H, S_{\mu}\right)$ and $\mathrm{S}_{\alpha}=\left(R_{\alpha}, H_{\alpha}, S_{1, \alpha}, \ldots, S_{n, \alpha}\right)$.

(iv) Return $\left\{\left(R_{\alpha}, H_{\alpha}, S_{\mu, \alpha}\right)\right\}_{\mu \in I}$, and add a pair $\left(M_{\alpha}, \mathrm{S}_{\alpha}\right)$ to the signature list.

(5-2) When $\mathcal{F}_{H}$ asks a pair $(M, R)$ of a message $M$ and $R \in M_{u}\left(\mathbb{Z}_{p}^{\times}\right)$to $\mathfrak{H}$, execute the following procedure:

(i) Increase $\alpha$ by one, and set $\left(M_{\alpha}, R_{\alpha}\right)=(M, R)$.

(ii) Check whether there exists an index $\xi=1, \ldots, \alpha-1$ such that $\left(M_{\alpha}, R_{\alpha}\right)=\left(M_{\xi}, R_{\xi}\right)$. If exists, set $H_{\alpha}=H_{\xi}$. Otherwise, choose $H_{\alpha} \in M_{u}\left(\mathbb{Z}_{p-1}\right)$ randomly.

(iii) Return $H_{\alpha}$. Then set $\mathrm{S}_{\alpha}=\left(R_{\alpha}, H_{\alpha}\right)$, and add a pair $\left(M_{\alpha}, \mathrm{S}_{\alpha}\right)$ to the signature list.

(6) If the following two conditions hold, then go to Step (7):

- $\mathcal{F}_{H}$ outputs a pair $\left(M^{\prime},\left(R^{\prime}, H^{\prime}, S^{\prime}\right)\right)$ of a message $M^{\prime} \notin \mathcal{M}_{\mathfrak{S}}$ and a valid signature $\left(R^{\prime}, H^{\prime}, S^{\prime}\right)$, and

- there exists an index $\alpha$ such that $\left(M^{\prime},\left(R^{\prime}, H^{\prime}\right)\right)=\left(M_{\alpha}, \mathrm{S}_{\alpha}\right)$.

Otherwise, output $\perp$, and halt.

(7) Clear the message list $\mathcal{M}_{\mathfrak{S}}$ and the subset $\left\{\left(M_{\xi}, \mathrm{S}_{\xi}\right)\right\}_{\xi \geq \alpha_{0}}$ of the signature list, where $\alpha_{0}$ is the minimum index which satisfies the second condition in Step (6). Then set $\alpha=0$, and run $\mathcal{F}_{H}$ on input $(p, g, A, Y)$ with the same random tape $\omega$ as Step (4).

(8) While running $\mathcal{F}_{H}$, if $\mathcal{F}_{H}$ queries $\mathfrak{S}$ or $\mathfrak{H}$, then go to Step (8-1) or (8-2).

(8-1) When $\mathcal{F}_{H}$ asks a pair $(M, I)$ to $\mathfrak{S}$, execute (i) in Step (5-1), and

- when $\alpha<\alpha_{0}$, return $\left\{\left(R_{\alpha}, H_{\alpha}, S_{\mu, \alpha}\right)\right\}_{\mu \in I}$ made in Step (5-1).

- when $\alpha>\alpha_{0}$, then execute (ii)-(iv) in Step (5-1).

(8-2) If $\mathcal{F}_{H}$ asks a pair $(M, R)$ to $\mathfrak{H}$, then execute (i) of Step (5-2), and

- when $\alpha<\alpha_{0}$, return $H_{\alpha}$ made in Step (5-2).

- when $\alpha=\alpha_{0}$, choose $H_{\alpha_{0}}^{\prime \prime} \in M_{u}\left(\mathbb{Z}_{p-1}\right)$ randomly with $H^{\prime}-H_{\alpha_{0}}^{\prime \prime} \in G L_{u}\left(\mathbb{Z}_{p-1}\right)$, and return $H_{\alpha_{0}}^{\prime \prime}$. Then set $\mathrm{S}_{\alpha_{0}}=\left(R_{\alpha_{0}}, H_{\alpha_{0}}^{\prime \prime}\right)$, and add $\left(M_{\alpha_{0}}, \mathrm{~S}_{\alpha_{0}}\right)$ to the signature list.

- when $\alpha>\alpha_{0}$, execute (ii) and (iii) in Step (5-2).

(9) If the following conditions hold, then go to Step (10):

- $\mathcal{F}_{H}$ outputs a pair $\left(M^{\prime \prime},\left(R^{\prime \prime}, H^{\prime \prime}, S^{\prime \prime}\right)\right)$ of a message $M^{\prime \prime} \notin \mathcal{M}_{\mathfrak{S}}$ and a valid signature $\left(R^{\prime \prime}, H^{\prime \prime}, S^{\prime \prime}\right)$, and

- $M^{\prime \prime}=M^{\prime}, R^{\prime \prime}=R^{\prime}$ and $H^{\prime \prime}=H_{\alpha_{0}}^{\prime \prime}$.

Otherwise, output $\perp$, and halt.

(10) Output $\left(S^{\prime}-S^{\prime \prime}\right)\left(H^{\prime}-H^{\prime \prime}\right)^{-1}$, and halt.

If the conditions in Steps (6) and (9) hold, then $\mathcal{A}_{H}$ succeeds in solving SP-HDLP since $X=\left(S^{\prime}-S^{\prime \prime}\right)\left(H^{\prime}-H^{\prime \prime}\right)^{-1}$ follows from the definiton of DHS.

Lemma 5.4. Let $P_{\mathcal{A}_{H}}$ be the success probability of $\mathcal{A}_{H}$. Then one has

$$
P_{\mathcal{A}_{H}} \geq \frac{3 \varepsilon^{2}}{400 q_{h}}
$$

We prove the lemma in $\$ 7$.

We see that Step (2) in $\mathcal{A}_{H}$ can be done in time $O\left(k^{3} u^{4}\right)$ since Procedure Find- $C$ takes time $O\left(k^{3} u^{4}\right)$. In Steps (5-1), (5-2), (8-1) and (8-2), for any query, one can construct a signature or a hash value in time $O\left(n k^{3} u^{3}\right)$. Since the 
inverse of $H^{\prime}-H^{\prime \prime}$ is computed in time $O\left(k^{3} u^{3}\right)$, Step (10) can be done in time $O\left(k^{3} u^{3}\right)$. Hence, the running time of $\mathcal{A}_{H}$ is given by

$$
2 t+2\left(q_{s}+q_{h}+1\right) O\left(n k^{3} u^{4}\right) .
$$

This proves (i) of Lemma A.

We next prove (ii) of Lemma A. Let $\mathcal{F}_{D}$ be a forger who breaks DDS with $\left(n, l ; t, q_{s}, q_{h}, \varepsilon\right)$. We construct a PPTM $\mathcal{A}_{D}$ as follows:

(1) $\mathcal{A}_{D}$ takes as input a tuple $(p, g, A, Y)$.

(2) Execute Procedure Find- $C$ on input $(p, g, A, Y)$, and set $X^{(2)}=C$.

(3) Set a counter $\alpha$ by 0 .

(4) Choose $X_{1}, \ldots, X_{n-1} \in M_{u}\left(\mathbb{Z}_{p-1}\right)$ randomly, and run $\mathcal{F}_{D}$ on input $(p, g, A, \tilde{Y})$ and $\left(X_{1}, \ldots, X_{l}\right)$ with some random tape $\omega$, where

$$
\tilde{Y}=\left(\begin{array}{cccc}
G^{X_{1}} & & & 1 \\
& \ddots & & \\
& & G^{X_{n-1}} & \\
1 & & & Y
\end{array}\right) .
$$

(5) While running $\mathcal{F}_{D}$, if $\mathcal{F}_{D}$ queries $\mathfrak{S}$ or $\mathfrak{H}$, then go to Step (5-1) or (5-2).

(5-1) When $\mathcal{F}_{D}$ asks a pair $(M, I)$ of a message $M$ and a subset $I \subset\{l+1, \ldots, n\}$ to $\mathfrak{S}$, execute the following procedure:

(i) Increase $\alpha$ by one, and set $M_{\alpha}=M$. Then add $M_{\alpha}$ to the message list $\mathcal{M}_{\mathfrak{S}}$.

(ii) For each $\mu=l+1, \ldots, n-1$, choose $H_{\mu} \in M_{u}\left(\mathbb{Z}_{p-1}\right)$ randomly, and compute $R_{\mu}$ and $S_{\mu}$ by using the algorithm $\wp_{D}$ and the matrices $X_{l+1}, \ldots, X_{n-1}$ chosen in Step (2).

(iii) Execute Procedure Simulation on input $\left(p, g, A, Y, X^{(2)}\right)$, and set $\left(R_{n}, H_{n}, S_{n}\right)=(R, H, S)$.

(iv) For each $\mu=l+1, \ldots, n$, set $\left(R_{\mu, \alpha}, H_{\mu, \alpha}, S_{\mu, \alpha}\right)=\left(R_{\mu}, H_{\mu}, S_{\mu}\right)$ and

$$
\mathrm{S}_{\alpha}=\left\{\left(R_{\mu, \alpha}, H_{\mu, \alpha}, S_{\mu, \alpha}\right)\right\}_{\mu=l+1}^{n} .
$$

(v) Return $\left\{\left(R_{\mu, \alpha}, H_{\mu, \alpha}, S_{\mu, \alpha}\right)\right\}_{\mu \in I}$, and add a pair $\left(M_{\alpha}, \mathrm{S}_{\alpha}\right)$ to the signature list.

(5-2) When $\mathcal{F}_{D}$ asks, to $\mathfrak{H}$, a pair $(M, \widetilde{R})$ of a message $M$ and $\widetilde{R} \in M_{n u}\left(\mathbb{Z}_{p}^{\times}\right)$of the form (3.1), execute the following procedure:

(i) Increase $\alpha$ by one, and set $\left(M_{\alpha}, R_{1, \alpha}, \ldots, R_{n, \alpha}\right)=\left(M, R_{1}, \ldots, R_{n}\right)$.

(ii) For each $\mu=1, \ldots, n$, check whether there exist an index $\xi=1, \ldots, \alpha-1$ such that $\left(M_{\alpha}, R_{\mu, \alpha}\right)=\left(M_{\xi}, R_{\mu, \xi}\right)$. If exists, set $H_{\mu, \alpha}=H_{\mu, \xi}$. Otherwise, choose $H_{\mu, \alpha} \in M_{u}\left(\mathbb{Z}_{p-1}\right)$ randomly.

(iii) Return

$$
\widetilde{H}_{\alpha}=\left(\begin{array}{ccc}
H_{1, \alpha} & & O \\
& \ddots & \\
O & & H_{n, \alpha}
\end{array}\right) .
$$

Then set $\mathrm{S}_{\alpha}=\left\{\left(R_{\mu, \alpha}, H_{\mu, \alpha}\right)\right\}_{\mu=1}^{n}$, and add a pair $\left(M_{\alpha}, \mathrm{S}_{\alpha}\right)$ to the signature list.

(6) If the following two conditions hold, then go to Step (7):

- $\mathcal{F}_{D}$ outputs a pair $\left(M^{\prime},\left(\widetilde{R^{\prime}}, \widetilde{H}^{\prime}, \widetilde{S}^{\prime}\right)\right)$ of a message $M^{\prime} \notin \mathcal{M}_{\mathfrak{S}}$ and a valid signature $\left(\widetilde{R}^{\prime}, \widetilde{H}^{\prime}, \widetilde{S}^{\prime}\right)$, and

- there exists an index $\alpha$ such that $M^{\prime}=M_{\alpha}, R_{n}^{\prime}=R_{n, \alpha}$ and $H_{n}^{\prime}=H_{n, \alpha}$.

Otherwise, output $\perp$, and halt.

(7) Clear the message list $\mathcal{M}_{\mathfrak{S}}$ and the subset $\left\{\left(M_{\xi}, \mathrm{S}_{\xi}\right)\right\}_{\xi>\alpha_{0}}$ of the signature list, where $\alpha_{0}$ is the minimum index which satisfies the second condition in Step (6). Then set $\alpha=0$, and run $\mathcal{F}_{D}$ on input $(p, g, A, \widetilde{Y})$ and $\left(X_{1}, \ldots, X_{l}\right)$ with the same random tape $\omega$ as Step (4).

(8) While running $\mathcal{F}_{D}$, if $\mathcal{F}_{D}$ queries $\mathfrak{S}$ or $\mathfrak{H}$, then go to Step (8-1) or (8-2).

(8-1) When $\mathcal{F}_{D}$ asks a pair $(M, I)$ to $\mathfrak{S}$, execute (i) of Step (5-1), and

- when $\alpha<\alpha_{0}$, return $\left\{\left(R_{\mu, \alpha}, H_{\mu, \alpha}, S_{\mu, \alpha}\right)\right\}_{\mu \in I}$ made in Step (5-1).

- when $\alpha>\alpha_{0}$, execute (ii)-(v) in Step (5-1).

(8-2) When $\widetilde{F}_{D}$ asks a pair $(M, \widetilde{R})$ to $\mathfrak{H}$, execute (i) of Step (5-2), and

- when $\alpha<\alpha_{0}$, return $\widetilde{H}_{\alpha}$ made in Step (5-2).

- when $\alpha=\alpha_{0}$, choose $H_{n, \alpha_{0}}^{\prime \prime} \in M_{u}\left(\mathbb{Z}_{p-1}\right)$ randomly with $H_{n}^{\prime}-H_{n, \alpha_{0}}^{\prime \prime} \in G L_{u}\left(\mathbb{Z}_{p-1}\right)$, and return 


$$
\widetilde{H}_{\alpha_{0}}^{\prime \prime}=\left(\begin{array}{cccc}
H_{1, \alpha_{0}} & & & O \\
& \ddots & & \\
& & H_{n-1, \alpha_{0}} & \\
O & & & H_{n, \alpha_{0}}^{\prime \prime}
\end{array}\right) .
$$

Then set $\mathrm{S}_{\alpha_{0}}=\left(\widetilde{\boldsymbol{R}}_{\alpha_{0}}, \widetilde{\boldsymbol{H}}_{\alpha_{0}}^{\prime \prime}\right)$, and add $\left(M_{\alpha_{0}}, \mathrm{~S}_{\alpha_{0}}\right)$ to the signature list.

- when $\alpha>\alpha_{0}$, execute (ii) and (iii) in Step (5-2).

(9) If the following conditions hold, then go to Step (10):

- $\mathcal{F}_{D}$ outputs a pair $\left(M^{\prime \prime},\left(\widetilde{R^{\prime \prime}}, \widetilde{H}^{\prime \prime},{\widetilde{S^{\prime \prime}}}^{\prime \prime}\right)\right.$ of a message $M^{\prime \prime} \notin \mathcal{M}_{\mathfrak{S}}$ and a signature $\left(\widetilde{R^{\prime \prime}}, \widetilde{H}^{\prime \prime},{\widetilde{S^{\prime \prime}}}^{\prime \prime}\right)$ which is valid, and - $M^{\prime \prime}=M^{\prime}, \widetilde{R}^{\prime \prime}=\widetilde{R}^{\prime}$ and $\widetilde{H}^{\prime \prime}=\widetilde{H}_{\alpha_{0}}^{\prime \prime}$ are satisfied.

Otherwise, output $\perp$, and halt.

(10) Output $\left(S_{n}^{\prime}-S_{n}^{\prime \prime}\right)\left(H_{n}^{\prime}-H_{n}^{\prime \prime}\right)^{-1}$, and halt.

It follows from the same argument as in the proof of (i) of Lemma A that the success probability of $\mathcal{A}_{D}$ is at least $\left(3 \varepsilon^{2}\right) /\left(400 q_{h}\right)$ and that the running time of $\mathscr{A}_{D}$ is given by

$$
2 t+2\left(q_{s}+q_{h}+1\right) O\left(n k^{3} u^{4}\right) \text {. }
$$

This completes the proof of (ii) of Lemma A.

\section{Indistinguishability of the Simulation}

We give the proof of Lemma 5.3 in this section. We use the same notation as in $\S 5$. Both $\Omega_{1}$ and $\Omega_{2}$ are of cardinality $(p-1)^{u^{2}(n+1)}$. This implies that

$$
d\left(\Omega_{1}, \Omega_{2}\right)=\sum_{\sigma \in \mathscr{S}_{1}} \frac{\left|N_{1, \sigma}-N_{2, \sigma}\right|}{(p-1)^{u^{2}(n+1)}},
$$

where $N_{i, \sigma}$ is the cardinality of the inverse image $F_{i}^{-1}(\{\sigma\})$ for $i=1,2$. Since $F_{1}$ is bijective, we have $N_{1, \sigma}=1$ for any $\sigma \in \mathscr{S}_{1}$.

Lemma 6.1. Let $\sigma=F_{1}\left(K_{1}, \ldots, K_{n}, H\right)=\left(R_{1}, \ldots, R_{n}, H, S_{1}, \ldots, S_{n}\right) \in \mathscr{S}_{1}$. Then $\sigma$ is also an element of $\mathscr{S}_{2}$ if and only if there exist matrices $E, V \in M_{u}\left(\mathbb{Z}_{q}\right)$ such that the following two equations hold:

$$
\begin{gathered}
2\left(E+X_{n} V\right)=K_{n} \bmod q, \\
H-2 V R^{*}=O \bmod q,
\end{gathered}
$$

where $R=R_{1} \otimes \cdots \otimes R_{n}$.

Proof. Assume that $\sigma \in \mathscr{S}_{2}$. Then there exist matrices $E_{n}, V_{n} \in M_{u}\left(\mathbb{Z}_{q}\right)$ and $W_{n}, H^{(2)} \in M_{u}\left(\mathbb{Z}_{2}\right)$ such that $K_{n}=2\left(E_{n}+X_{n} V_{n}\right)+q W_{n} \bmod p-1$ and $H=2 V_{n} R^{*}+q H^{(2)} \bmod p-1$. Setting $E=E_{n}$ and $V=V_{n}$, we see that $E$ and $V$ satisfy the Eqs. (6.1) and (6.2) by the definition of $\mathscr{S}_{2}$.

On the other hand, assume that there exist matrices $E, V \in M_{u}\left(\mathbb{Z}_{q}\right)$ such that the Eqs. (6.1) and (6.2) hold. Set $E_{n}=E, V_{n}=V, H^{(2)}=H \bmod 2, W_{n}=K_{n} \bmod 2$ and

$$
\sigma^{\prime}=F_{2}\left(K_{1}, \ldots, K_{n-1}, E_{n}, V_{n}, W_{n}, H^{(2)}\right)=\left(G^{K_{1}}, \ldots, G^{K_{n-1}}, R_{n}^{\prime}, H^{\prime}, S_{1}^{\prime}, \ldots, S_{n}^{\prime}\right) .
$$

We show that $\sigma^{\prime}=\sigma$. Note that $R_{n}^{\prime}=G^{K_{n}^{\prime}}=G^{2\left(E_{n}+X_{n} V_{n}\right)+q W_{n}}$. Since $K_{n} \bmod q=2\left(E_{n}+X_{n} V_{n}\right)$ and $K_{n} \bmod 2=$ $W_{n} \bmod 2=q W_{n} \bmod 2$, we have $K_{n}=K_{n}^{\prime} \bmod p-1$. This shows that $R_{n}=R_{n}^{\prime}$. By the Eqs. (6.1) and (6.2) and the definition of $F_{2}$, we have $H=H^{\prime} \bmod p-1$ and $S_{n}=S_{n}^{\prime} \bmod p-1$. Hence $\sigma=\sigma^{\prime} \in \mathscr{S}_{2}$ follows.

The proof of Lemma 6.1 implies that $N_{2, \sigma}$ is equal to the number of solutions $(E, V)$ of the Eqs. (6.1) and (6.2). Since, for any given matrix $V, E$ is uniquely determined by (6.1), we see that $N_{2, \sigma}$ is equal to the number of solutions $V$ of (6.2). We now evaluate $N_{2, \sigma}$. We write $R^{*(q)}=R^{*} \bmod q$ and $H^{(q)}=H \bmod q$. The Eq. (6.2) can be rewritten in the form

$$
H^{(q)}-2 V R^{*(q)}=O .
$$

Fix an index $i$, and we consider a system of linear equation

$$
h_{i}-2 v_{i} R^{*(q)}=0
$$

where $h_{i}$ and $v_{i}$ are the $i$-th row vectors of $H^{(q)}$ and $V^{(q)}$, respectively. The system (6.4) has a solution if and only if $h_{i}$ can be written in a linear combination of $r_{1}, \ldots, r_{u}$, where $r_{i}$ denotes the $i$-th row vector of $R^{*(q)}$. In addition, if the system (6.4) has a solution $v_{0}$, then all the solutions are of the form $v_{0}+v$, where $v$ is an element of the solution space $U_{i}$ of the system $2 v_{i} R^{*(q)}=0$. We also see that the dimension of $U_{i}$ is $u-r$, where $r$ is the rank of $R^{*(q)}$. This implies that the number of solutions of the system (6.4) is $q^{u-r}$ if the solutions exist. Hence we have the following propositions: 
Proposition 6.2. Assume that $R^{*(q)}$ is of rank $r$.

(i) The number of solutions of (6.3) is $q^{u(u-r)}$ if the solutions exist.

(ii) The cardinality of the set

$$
\mathscr{H}_{R^{*(q)}}=\left\{H^{(q)} \in M_{u}\left(\mathbb{Z}_{q}\right) \mid(6.3) \text { has a solution. }\right\}
$$

is $q^{r u}$.

Proposition 6.3. Let $\sigma=\left(R_{1}, \ldots, R_{n}, H, S_{1}, \ldots, S_{n}\right) \in \mathscr{S}_{1}$, and assume that the matrix $R^{*(q)}$ is of rank $r$. Then

$$
N_{2, \sigma}= \begin{cases}q^{u(u-r)} & \text { if } H^{(q)} \in \mathscr{H}_{R^{*(q)}}, \\ 0 & \text { otherwise. }\end{cases}
$$

In order to compute $d\left(\Omega_{1}, \Omega_{2}\right)$, we need to evaluate $C(r, u, q)$, the number of matrices of rank $r$ in $M_{u}\left(\mathbb{Z}_{q}\right)$. The following proposition holds:

Proposition 6.4. For $1 \leq r \leq u$,

$$
C(r, u, q)=\frac{\left\{\left(q^{u}-1\right) \cdots\left(q^{u}-q^{r-1}\right)\right\}^{2}}{\left(q^{r}-1\right) \cdots\left(q^{r}-q^{r-1}\right)}
$$

and $C(0, u, q)=1$

The proof of this proposition is implicitly given in the proof of Theorem 9.5.2 of [5]. The formula $k_{j}$ in the proof of the theorem gives the expression of $C(r, u, q)$ if we substitute $d=e=u$ and $j=r$ for $k_{j}$.

Using this proposition, we have

$$
\begin{aligned}
d\left(\Omega_{1}, \Omega_{2}\right) & =\frac{2^{u^{2}(n+1)} q^{u^{2}(n-1)}}{(p-1)^{u^{2}(n+1)}} \sum_{r=0}^{u} C(r, u, q)\left\{q^{u r}\left(q^{u(u-r)}-1\right)+\left(q^{u^{2}}-q^{u r}\right)(1-0)\right\} \\
& \leq \frac{2}{q^{u^{2}}}+\frac{2}{q^{2 u^{2}}} \sum_{r=1}^{u-1} C(r, u, q)\left(q^{u^{2}}-q^{u r}\right) \\
& \leq \frac{2}{q}+\sum_{r=1}^{u-1} \frac{2}{q\left(1-q^{-1}\right)^{r}}<\frac{2 u}{q-u+1} .
\end{aligned}
$$

Since $p=2 q+1$ and $p$ is of $k$ bits long, we have $2^{k-2}<q$, which implies that $d\left(\Omega_{1}, \Omega_{2}\right)$ is negligible in $k$.

\section{The Success Probability of $\mathscr{A}_{H}$}

We prove Lemma 5.4 in this section.

We assume that $q_{s}=0$. Let $\mathbb{O}_{k}$ be the set of the binary strings of length $t(k)$ which are written in the random tape of $\mathcal{F}_{H}$, and set $\mathbb{H}_{k}=\left(M_{u}\left(\mathbb{Z}_{p-1}\right)\right)^{q_{h}(k)}$. For $\omega \in \mathbb{O}_{k}$ and $\boldsymbol{H}=\left(H_{1}, \ldots, H_{q_{h}(k)}\right) \in \mathbb{H}_{k}, \mathcal{F}_{H}^{\omega, \boldsymbol{H}}$ denotes the deterministic machine obtained from $\mathcal{F}_{H}$, where the computation in $\mathcal{F}_{H}$ is specified by the string $\omega$ in the random tape, and for the $\alpha$-th query $\left(M_{\alpha}, R_{\alpha}\right)$ to the oracle $\mathfrak{H}$, the $\alpha$-th entry $H_{\alpha}$ of $\boldsymbol{H}$ is returned as the answer. By the definition of $\mathcal{F}_{H}$, we have

$$
\varepsilon=\operatorname{Pr}_{(\omega, \boldsymbol{H}) \in \mathbb{O}_{k} \times \mathbb{H}_{k}}\left[\mathcal{F}_{H}^{\omega, \boldsymbol{H}} \text { succeeds }\right] .
$$

For each $\alpha=1, \ldots, q_{h}(k)$, let $\mathbb{S}_{\alpha}$ be the set of elements $(\omega, \boldsymbol{H}) \in \mathbb{O}_{k} \times \mathbb{H}_{k}$ which satisfy the following three conditions:

(i) $\mathcal{F}_{H}^{\omega, \boldsymbol{H}}$ succeeds, that is, $\mathcal{F}_{H}^{\omega, \boldsymbol{H}}$ outputs a valid pair $(M,(R, H, S))$ with $M \notin \mathcal{M}_{\mathfrak{S}}$,

(ii) $(M, R, H)=\left(M_{\alpha}, R_{\alpha}, H_{\alpha}\right)$, and

(iii) $(M, R, H) \neq\left(M_{\beta}, R_{\beta}, H_{\beta}\right)$ for any $\beta=1, \ldots, \alpha-1$.

We then define

$$
\mathbb{H}_{k}(\boldsymbol{H}, \beta)=\left\{\widehat{\boldsymbol{H}} \in \mathbb{H}_{k} \mid\left(H_{1}, \ldots, H_{\beta}\right)=\left(\widehat{H}_{1}, \ldots, \widehat{H}_{\beta}\right)\right\}
$$

and $\mathbb{H}_{k}(\boldsymbol{H}, 0)=\mathbb{H}_{k}$ for $\boldsymbol{H} \in \mathbb{H}_{k}$ and $\beta=1, \ldots, q_{h}(k)-1$. Since $\mathbb{S}_{\alpha_{1}} \cap \mathbb{S}_{\alpha_{2}}=\emptyset$ for any $\alpha_{1} \neq \alpha_{2}$, using these sets, one can see that

where

$$
P_{\mathcal{A}_{H}}=\sum_{\alpha=1}^{q_{h}(k)} \operatorname{Pr}_{(\omega, \boldsymbol{H}, \widehat{\boldsymbol{H}}) \in T_{\alpha}}\left[(\omega, \boldsymbol{H}) \in \mathbb{S}_{\alpha},(\omega, \widehat{\boldsymbol{H}}) \in \mathbb{S}_{\alpha} \text { and } H_{\alpha} \neq \widehat{\boldsymbol{H}}_{\alpha}\right]
$$

$$
T_{\alpha}=\left\{(\omega, \boldsymbol{H}, \widehat{\boldsymbol{H}}) \in \mathbb{O}_{k} \times \mathbb{H}_{k} \times \mathbb{H}_{k} \mid \widehat{\boldsymbol{H}} \in \mathbb{H}_{k}(\boldsymbol{H}, \alpha-1)\right\} .
$$

We can apply the argument in the proof of Lemma 2 of [7] since $\varepsilon \geq 7 q_{h} / 2^{k}$. Using that, we see the following facts: 
(i) One has

$$
\operatorname{Pr}_{(\omega, \boldsymbol{H}) \in \mathbb{O}_{k} \times \mathbb{H}_{k}}[(\omega, \boldsymbol{H}) \in \mathbb{S}] \geq \varepsilon-\frac{1}{2^{k}} \geq \frac{6}{7} \varepsilon
$$

where $\mathbb{S}=\mathbb{S}_{1} \cup \cdots \cup \mathbb{S}_{q_{h}(k)}$.

(ii) Set

$$
J=\left\{\alpha \mid \operatorname{Pr}_{(\omega, \boldsymbol{H}) \in \mathbb{O}_{k} \times \mathbb{H}_{k}}\left[(\omega, \boldsymbol{H}) \in \mathbb{S}_{\alpha} \mid(\omega, \boldsymbol{H}) \in \mathbb{S}\right] \geq \frac{1}{2 q_{h}(k)}\right\},
$$

and for each $\alpha \in J$, let $\mathbb{O}_{\alpha}^{\prime}$ be the set of elements $(\omega, \boldsymbol{H}) \in \mathbb{O}_{k} \times \mathbb{H}_{k}$ which satisfy the following inequality:

$$
\begin{aligned}
\operatorname{Pr}_{\widehat{\boldsymbol{H}} \in \mathbb{H}_{k}}\left[(\omega, \widehat{\boldsymbol{H}}) \in \mathbb{S}_{\alpha} \mid \widehat{\boldsymbol{H}} \in \mathbb{H}_{k}(\boldsymbol{H}, \alpha-1)\right] & =\underset{\widehat{\boldsymbol{H}} \in \mathbb{H}_{k}(\boldsymbol{H}, \alpha-1)}{\operatorname{Pr}}\left[(\omega, \widehat{\boldsymbol{H}}) \in \mathbb{S}_{\alpha}\right] \\
& \geq \frac{3 \varepsilon}{14 q_{h}(k)} .
\end{aligned}
$$

Then one has

$$
\operatorname{Pr}_{(\omega, \boldsymbol{H}) \in \mathbb{O}_{k} \times \mathbb{H}_{k}}\left[(\omega, \boldsymbol{H}) \in \mathbb{O}_{\alpha}^{\prime} \mid(\omega, \boldsymbol{H}) \in \mathbb{S}_{\alpha}\right] \geq \frac{1}{2} .
$$

It follows from the Eqs. (7.2) and (7.4) that

$$
\begin{aligned}
& \operatorname{Pr} \operatorname{Pr}_{(\omega, \boldsymbol{H}) \in \mathbb{O}_{k} \times \mathbb{H}_{k}}\left[(\omega, \boldsymbol{H}) \in \mathbb{O}_{\alpha}^{\prime} \cap \mathbb{S}_{\alpha}\right] \\
= & \operatorname{Pr}_{(\omega, \boldsymbol{H}) \in \mathbb{O}_{k} \times \mathbb{H}_{k}}\left[(\omega, \boldsymbol{H}) \in \mathbb{O}_{\alpha}^{\prime} \mid(\omega, \boldsymbol{H}) \in \mathbb{S}_{\alpha}\right] \underset{(\omega, \boldsymbol{H}) \in \mathbb{O}_{k} \times \mathbb{H}_{k}}{\operatorname{Pr}}\left[(\omega, \boldsymbol{H}) \in \mathbb{S}_{\alpha}\right] \\
\geq & \frac{1}{2} \operatorname{Pr}_{(\omega, \boldsymbol{H}) \in \mathbb{O}_{k} \times \mathbb{H}_{k}}\left[(\omega, \boldsymbol{H}) \in \mathbb{S}_{\alpha} \mid(\omega, \boldsymbol{H}) \in \mathbb{S}\right] \underset{(\omega, \boldsymbol{H}) \in \mathbb{O}_{k} \times \mathbb{H}_{k}}{\operatorname{Pr}}[(\omega, \boldsymbol{H}) \in \mathbb{S}] \\
\geq & \frac{3}{7} \varepsilon \operatorname{Pr}_{(\omega, \boldsymbol{H}) \in \mathbb{O}_{k} \times \mathbb{H}_{k}}\left[(\omega, \boldsymbol{H}) \in \mathbb{S}_{\alpha} \mid(\omega, \boldsymbol{H}) \in \mathbb{S}\right] .
\end{aligned}
$$

Hence, we have

$$
\begin{aligned}
P_{\mathcal{A}_{H}} \geq & \sum_{\alpha \in J} \underset{(\omega, \boldsymbol{H}, \widehat{\boldsymbol{H}}) \in T_{\alpha}}{\operatorname{Pr}}\left[(\omega, \boldsymbol{H}) \in \mathbb{S}_{\alpha} \cap \mathbb{O}_{\alpha}^{\prime},(\omega, \widehat{\boldsymbol{H}}) \in \mathbb{S}_{\alpha} \text { and } H_{\alpha} \neq \widehat{\boldsymbol{H}}_{\alpha}\right] \\
\geq & \sum_{\alpha \in J} \underset{(\omega, \boldsymbol{H}, \widehat{\boldsymbol{H}}) \in T_{\alpha}}{\operatorname{Pr}}\left[(\omega, \boldsymbol{H}) \in \mathbb{O}_{\alpha}^{\prime} \cap \mathbb{S}_{\alpha}\right] \\
& \times \underset{(\omega, \boldsymbol{H}, \widehat{\boldsymbol{H}}) \in T_{\alpha}}{\operatorname{Pr}}\left[(\omega, \widehat{\boldsymbol{H}}) \in \mathbb{S}_{\alpha} \text { and } H_{\alpha} \neq \widehat{\boldsymbol{H}}_{\alpha} \mid(\omega, \boldsymbol{H}) \in \mathbb{S}_{\alpha} \cap \mathbb{O}_{\alpha}^{\prime}\right] \\
\geq & \frac{3}{7} \varepsilon \sum_{\alpha \in J} \operatorname{Pr}_{(\omega, \boldsymbol{H}) \in \mathbb{O}_{k} \times \mathbb{H}_{k}}\left[(\omega, \boldsymbol{H}) \in \mathbb{S}_{\alpha} \mid(\omega, \boldsymbol{H}) \in \mathbb{S}\right] \\
& \times \underset{(\omega, \boldsymbol{H}, \widehat{\boldsymbol{H}}) \in T_{\alpha}^{\prime}}{\operatorname{Pr}}\left[(\omega, \widehat{\boldsymbol{H}}) \in \mathbb{S}_{\alpha} \text { and } H_{\alpha} \neq \widehat{\boldsymbol{H}}_{\alpha}\right],
\end{aligned}
$$

where

$$
T_{\alpha}^{\prime}=\left\{(\omega, \boldsymbol{H}, \widehat{\boldsymbol{H}}) \in T_{\alpha} \mid(\omega, \boldsymbol{H}) \in \mathbb{S}_{\alpha} \cap \mathbb{O}_{\alpha}^{\prime}\right\} .
$$

Using the proof of Lemma 2 and the Eq. (7.3), for any $\alpha \in J$ and $(\omega, \boldsymbol{H}) \in \mathbb{O}_{\alpha}^{\prime} \cap \mathbb{S}_{\alpha}$, we have

$$
\begin{aligned}
& \quad \underset{\widehat{\boldsymbol{H}} \in \mathbb{H}_{k}(\boldsymbol{H}, \alpha-1)}{\operatorname{Pr}}\left[(\omega, \widehat{\boldsymbol{H}}) \in \mathbb{S}_{\alpha} \text { and } H_{\alpha} \neq \widehat{\boldsymbol{H}}_{\alpha}\right] \\
& \geq \underset{\widehat{\boldsymbol{H}} \in \mathbb{H}_{k}(\boldsymbol{H}, \alpha-1)}{\operatorname{Pr}}\left[(\omega, \widehat{\boldsymbol{H}}) \in \mathbb{S}_{\alpha}\right]-\underset{\widehat{\boldsymbol{H}} \in \mathbb{H}_{k}(\boldsymbol{H}, \alpha-1)}{\operatorname{Pr}}\left[(\omega, \widehat{\boldsymbol{H}}) \in \mathbb{S}_{\alpha} \text { and } \widehat{H}_{\alpha}=H_{\alpha}\right] \\
& \geq \frac{3 \varepsilon}{14 q_{h}(k)}-\underset{\widehat{\boldsymbol{H}} \in \mathbb{H}_{k}(\boldsymbol{H}, \alpha-1)}{\operatorname{Pr}}\left[\widehat{H}_{\alpha}=H_{\alpha}\right] \geq \frac{3 \varepsilon}{14 q_{h}(k)}-\frac{1}{2^{k}} \geq \frac{\varepsilon}{14 q_{h}(k)} .
\end{aligned}
$$

By Lemma 3 of [7], we obtain

$$
\begin{aligned}
P_{\mathcal{A}_{H}} & \geq \frac{3 \varepsilon^{2}}{98 q_{h}(k)} \sum_{\alpha \in J} \operatorname{Pr}_{(\omega, \boldsymbol{H}) \in \mathbb{O}_{k} \times \mathbb{H}_{k}}\left[(\omega, \boldsymbol{H}) \in \mathbb{S}_{\alpha} \mid(\omega, \boldsymbol{H}) \in \mathbb{S}\right] \\
& \geq \frac{3 \varepsilon^{2}}{98 q_{h}(k)} \cdot \frac{1}{2}=\frac{3 \varepsilon^{2}}{196 q_{h}(k)} .
\end{aligned}
$$

We now assume that $q_{s}>0$. One can apply the argument in the proof of Lemma 4 of [7] by the assumption of Lemma A. If one replaces the signer oracle with Procedure Simulation, then there exists some risk of collisions. 
The proof of Lemma 4 insists that the probability that $\mathcal{F}_{H}$ succeeds with no collision is at least $7 \varepsilon / 10 \geq 7 q_{h} / 2^{k}$ and that one can apply the argument in the proof of Lemma 2 by replacing $\varepsilon$ with $\widetilde{\varepsilon}=7 \varepsilon / 10$. Hence, by the Eq. (7.5), we see that

$$
P_{\mathcal{A}_{H}} \geq \frac{3 \widetilde{\varepsilon}^{2}}{196 q_{h}(k)}=\frac{3 \varepsilon^{2}}{400 q_{h}(k)}
$$

proving the lemma.

\section{Concluding Remarks}

We have proposed two distributed signature schemes, the distributed hyper-powering signature scheme (DHS) and the distributed diagonal hyper-powering signature scheme (DDS), and obtained the following security results under the SP-HDLP assumption:

- DHS is $(n, 0)$-EUF-ACMA, and

- DDS is $(n, l)$-EUF-ACMA for any $0 \leq l \leq n-1$.

This implies that one can apply these signature schemes to a strategy for recovering PKIs.

Note that there is a difference between the security results of DHS and DDS. We naturally wonder whether DHS is $(n, l)$-EUF-ACMA for any $0 \leq l \leq n-1$. However, this question remains unsolved in this paper. There are two reasons for this.

One is the difference of the signing algorithms. In DDS, each CA can independently construct its partial signature. On the other hand, in DHS, all the CAs need common information about $R$ and $H$ in order to make partial signatures. Hence, if a part of the private key has been lost, then each CA cannot construct its partial signature by itself.

One of the methods for avoiding this is to change the specification of the forger so that the forger and each CA may cooperate. In this situation, when matrices $R_{1}, \ldots, R_{l}$ are given by the forger, we need to solve efficiently the equation $R_{1} \otimes \cdots \otimes R_{l}=G^{X^{\prime}}$ with respect to $X^{\prime}$. However, it is hard to do that under the SP-HDLP assumption.

The other reason is resistance to the rogue-key attack. As stated in $\S 3$, DHS is vulnerable to this attack. Namely, DHS is $(n, l)$-existentially forgeable for any $1 \leq l \leq n-1$ if one admits the forger to do the attack. On the other hand, DDS is not vulnerable to this attack. Hence, one can naturally expect that a general distributed signature scheme $\Sigma$ is resistant to the rogue-key attack if and only if, for any $0 \leq l \leq n-1, \Sigma$ is $(n, l)$-EUF-ACMA in our forger model. However, it is not known whether the statement holds or not.

\section{Acknowledgment}

The authors would like to thank Yuichi Futa and Manabu Maeda for their helpful suggestion. The authors would also thank referees for their helpful comments and suggestions.

\section{REFERENCES}

[1] Agrawal, M., Kayal, N., and Saxena, N., "PRIMES is in P," Ann. of Math. (2), 160: 781-793 (2004).

[2] Bellare, M., and Neven, G., "Multi-signatures in the plain public-key model and a general forking lemma," Proc. CCC '06, 390-399 (2006).

[3] Bellare, M., and Rogeway, P., "Random oracles are practical: A paradigm for designing efficient protocols," Proc. of the First CCCS, 62-73, ACM Press, New York (1993).

[4] Bellare, M., and Rogeway, P., "The exact security of digital signatures - How to sign with RSA and Rabin," Eurocrypt '96, LNCS 1070, 399-416, Springer-Verlag, Berlin (1996).

[5] Brouwer, A. E., Cohen, A. M., and Neumaier, A., Distance-Regular Graphs, Springer-Verlag (1989).

[6] Harn, L., "Group oriented $(t, n)$ threshold digital signature scheme and digital multisignature," IEE Proc.-Computers and Digital Techniques, 141: 307-313 (1994).

[7] Pointcheval, D., and Stern, J., "Security arguments for digital signatures and blind signatures," J. of Cryptology, 13: 361-396 (2000).

[8] Schnorr, C. P., "Efficient signature generation by smart card," J. of Cryptology, 4: 161-174 (1991).

[9] Shizuya, H., "Zero-knowledge interactive proofs for hyper- and elliptic-discrete logarithm problems," Proc. 1989 Workshop on Cryptography and Information Security, 143-152 (1989).

[10] Yoshida, M., Hasegawa, S., Isobe, S., Koizumi, E., Sakai, M., Shizuya, H., Futa, Y., and Maeda, M., "A technique for making PKI robust (in Japanese)," Technical Report of IEICE, Vol. 106, No. 597: 119-124 (2007). 